\title{
Saccadic Eye Movements in Elderly Depressed Patients With Suicidal Behaviors: An Exploratory Eye-Tracking Study
}

\author{
Yoan Barsznica ${ }^{1,2,3,4 *}$, Nicolas Noiret ${ }^{5}$, Bérénice Lambert ${ }^{6}$, Julie Monnin 1,3,4, \\ Claire De Pinho', Julia Hickel', Caroline Masse ${ }^{1,3}$, Stephane Richard-Devantoy, \\ Cynthia Morgny ${ }^{6}$, Djamila Bennabi1,3, Emmanuel Haffen ${ }^{1,3}$, Eric Laurent ${ }^{3}$, \\ Pierre Vande $1^{1,3,4,6}$ and Gilles Chopard ${ }^{1,2,3,4}$
}

${ }^{1}$ Department of Clinical Psychiatry, Besançon University Hospital, Besançon, France, ${ }^{2}$ Department of Neurology, Besançon University Hospital, Besançon, France, ${ }^{3}$ Laboratory of Neurosciences and Cognitive Psychology, University of Bourgogne Franche-Comté, Besançon, France, ${ }^{4}$ Memory Center of Research and Resources (CMRR), Besançon University Hospital, Besançon, France, ${ }^{5}$ UMR CNRS 7295 "Research Centre on Cognition and Learning," University of Poitiers, Poitiers, France,

OPEN ACCESS

Edited by:

Jun Gao,

Southwest University, China

Reviewed by: Niharika Singh,

University of Allahabad, India

Dongsheng Zhou,

Ningbo Kangning Hospital, China

${ }^{*}$ Correspondence:

Yoan Barsznica

ybarsznica@chu-besancon.fr

Specialty section:

This article was submitted to

Psychopathology,

a section of the journal

Frontiers in Psychology

Received: 20 May 2021 Accepted: 11 October 2021

Published: 11 November 2021

Citation:

Barsznica Y, Noiret N, Lambert B, Monnin J, De Pinho C, Hickel J,

Masse C, Richard-Devantoy S,

Morgny $C$, Bennabi D, Haffen $E$, Laurent $E$, Vandel $P$ and Chopard $G$ (2021) Saccadic Eye Movements in

Elderly Depressed Patients With Suicidal Behaviors: An Exploratory Eye-Tracking Study.

Front. Psychol. 12:712347. doi: 10.3389/fpsyg.2021.712347
${ }^{6}$ Regional Health Observatory, Bourgogne-Franche-Comté, France, ${ }^{7}$ Department of Old Age Psychiatry, Association Hospitalière de Bourgogne Franche-Comté Hospital, Bavilliers, France, ${ }^{8}$ McGill Group for Suicide Studies, Douglas Mental Health University Institute, Department of Psychiatry, McGill University, Montreal, QC, Canada

Suicidal behaviors (SBs) are often associated with impaired performance on neuropsychological executive functioning (EF) measures that encourage the development of more specific and reliable tools. Recent evidence could suggest that saccadic movement using eye tracking can provide reliable information on EF in depressive elderly. The aim of this study was to describe oculomotor performances in elderly depressed patients with SB. To achieve this aim, we compared saccadic eye movement (SEM) performances in elderly depressed patients $(N=24)$ with SB and with no SB in prosaccade (PS) and antisaccade (AS) tasks under the gap, step, and overlap conditions. All participants also underwent a complete neuropsychological battery. Performances were impaired in patients with SB who exhibited less corrected AS errors and longer time to correct them than patients with no SB. Moreover, both groups had a similar performance for PS latencies and correct AS. These preliminary results suggested higher cognitive inflexibility in suicidal patients compared to nonsuicidal. This inflexibility may explain the difficulty of the depressed elderly in generating solutions to the resurgence of suicidal ideation (SI) to respond adequately to stressful environments. The assessment of eye movement parameters in depressed elderly patients may be a first step in identifying high-risk patients for suicide.

Keywords: depression, elderly suicide, executive function, eye movement analysis, eye tracking

Abbreviations: AS, Antisaccade; AVF, Alternate Verbal Fluency; C-SSRS, Columbia Suicide Severity Rating Scale; C/W, Stroop color interference; EF, executive function; MMSE, Mini-Mental State Examination; PS, Prosaccade; PVF, Phonemic Verbal Fluency; SA, Suicide Attempt; SB, Suicidal Behaviors; SEM, saccadic eye movements; SI, Suicidal Ideation; SVF, Semantic Verbal Fluency; TMT, Trail Making Test. 


\section{INTRODUCTION}

Around one million people die by suicide and ten million people make a suicide attempt (SA) each year worldwide (World Health Organization, 2014). Suicide is therefore a major public health problem, especially among the elderly population (Shah et al., 2016). The ratio between SA and completed suicide has been estimated to be 4:1 in the elderly versus 200:1 in young adults (De Leo et al., 2001; Conwell and Thompson, 2008). Additionally, suicide is often associated with unipolar depression among the elderly (Alexopoulos et al., 1999; Szanto et al., 2001). Thus, agerelated suicide vulnerability associated with depression requires great vigilance (Bazin, 2004) and emphasizes the need to identify high-risk patients.

Age-related suicide vulnerability could be explained by cognitive and emotional inability to respond adequately to stressful environmental factors (Richard-Devantoy et al., 2012). In accordance with this proposal, executive function (EF) seems to play a crucial role in the suicide vulnerability in aging people in that it refers to abilities required to facilitate adaptation to novel and/or complex situations (Jurado and Rosselli, 2007). Elderly depressed patients with a history of SA or suicidal ideation (SI) are found to have poorer executive performance than nonsuicidal depressed and healthy elderly especially on tasks that rely on inhibitory control and cognitive flexibility (King et al., 2000; Marzuk et al., 2005; Westheide et al., 2008; Mcgirr et al., 2012; Richard-Devantoy et al., 2012, 2015, 2016).

From a clinical perspective, the neuropsychological tests measuring EFs, such as the Trail Making Test (TMT) B (Richard-Devantoy et al., 2012) or the Stroop test (Stroop, 1935; Meulemans, 2008), might be used as a potential predictive indicator of the risk of suicidal behavior (SB). However, the neuropsychological tests do not provide a pure measure of $\mathrm{EF}$ and include other non-executive processes which make validation difficult (Miyake et al., 2000). Furthermore, it is well established that an isolated low score may have little relevance in clinical evaluation (Iverson et al., 2011). Therefore, it is still too early to use neuropsychological tests as predictors of SB (RichardDevantoy et al., 2013). This should encourage the development of more specific and reliable tools.

Another approach for assessing executive impairment is based on oculomotor measurements. Eye-tracking tasks allow more detailed analysis and limiting the measurement bias of neuropsychological tests (for example, launching the task, timing, language, or motor difficulties). They are simple, short, and easily understandable for patients. Among the different tasks used in eye tracking are the prosaccade (PS) and antisaccade (AS) tasks (Leigh and Kennard, 2004; Hutton and Ettinger, 2006). In the PS task, participants are typically instructed to look from a central fixation dot toward a sudden onset peripheral target dot as quickly as possible. Although PS latency usually serves as a measure of processing speed (Carvalho et al., 2014; Noiret et al., 2017), it is also used to investigate the process of disengagement of attention by introducing gap periods between the disappearance of the central fixation dot and the appearance of the target dot (known as gap and overlap saccadic paradigms) (Saslow, 1967;
Fischer and Boch, 1983; Kalesnykas and Hallett, 1987; Braun and Breitmeyer, 1988; Fischer et al., 1993). In the gap condition, attention is not focused on the central dot when the target appears, resulting in faster saccadic engagement on the target, and so faster saccadic latency than overlap conditions (Pratt et al., 2006). In AS task, participants are instructed to refrain from looking at the peripheral target dot and direct their gaze in the opposite direction. The AS task is used to investigate the ability to inhibit saccades toward the stimulus and to correct potential saccade errors (i.e., saccade toward the peripheral target dot). In this sense, AS measures (e.g., latency, proportions of AS correct and corrected, and the time to correct AS errors) may reliably inform on EF.

Although many studies have used PS and AS tasks in several psychiatric and neurological conditions (Everling and Fischer, 1998; Hutton and Ettinger, 2006; Armstrong and Olatunji, 2012; Carvalho et al., 2014; Levy et al., 2018), few have examined saccadic eye movement (SEM) performances in normal aging or in elderly depression. Noiret et al. (2017) reported that latencies, time to correct AS errors, and proportion of uncorrected AS increased with aging. Elderly depressed patients were found to have longer latency in PS and AS tasks and a higher proportion of uncorrected saccades in AS task (Carvalho et al., 2014).

However, to our knowledge, no information is available concerning oculomotor measurement in elderly depressed patients with SB (Barsznica et al., 2019). The main objective of our research was to describe oculomotor performances in elderly depressed patients with SB. In this exploratory study, we expected these patients would have lower performances in these SEM tasks compared to elderly depressed patients without SB because of poorer EF that was previously reported compared to other populations (King et al., 2000; Marzuk et al., 2005; Westheide et al., 2008; Mcgirr et al., 2012; Richard-Devantoy et al., 2012, 2015). The use of standard overlap and gap conditions is to measure selective attention (Saslow, 1967; Fischer and Boch, 1983; Kalesnykas and Hallett, 1987; Braun and Breitmeyer, 1988; Fischer et al., 1993).

\section{MATERIALS AND METHODS}

\section{Population}

Thirty-two inpatients aged from 65 to 86 years were recruited in Nord Franche-Comté Hospital, Bavilliers, France. All patients were interviewed by a trained psychiatrist and presented a current major depressive episode according to diagnostic and statistical manual of mental disorders (DSM)-5 criteria (American Psychiatric Association, 2013) and a Montgomery-Åsberg Depression Rating Scale score $>20$ (MADRS) (Montgomery and Asberg, 1979). The patient with no SB group $(n=12)$ did not present the personal history of SA or recurring SI, according to the Columbia Suicide Severity Rating Scale (CSSRS; Posner et al., 2008). The patient with SB group $(n=12)$ presented a recent history of SA or recurring SI according to the CSSRS. Any patient with a previous medical history of a neurological disease (i.e., head trauma, stroke, dementia, Parkinson's disease, epilepsy, or brain tumor) or a psychiatric 
disorder (i.e., schizophrenia, bipolar disorder, and addictive behaviors other than smoking or borderline personality) was not included. All patients had a normal or corrected-to-normal vision and reported no visual disorders.

All patients were taking antidepressant medications at the time of testing. Informed written consent was obtained from all participants prior to enrollment. The research was approved by the Committee for the Protection of Persons and was conducted in accordance with the Declaration of Helsinki as revised in 1989.

\section{Neuropsychological Assessment}

All patients underwent a neuropsychological assessment protocol focused on the assessment of attention/processing speed, EFs, and verbal episodic memory. General cognitive function was assessed by Mini-Mental State Examination (MMSE; Folstein et al., 1975; Kalafat et al., 2003). This battery of tests was designed to ensure that no participant had cognitive impairments associated with dementia.

\section{Assessment of Attention and Processing Speed}

The TMT, part A (Reitan, 1958; Godefroy, 2008): Participants are required to connect with lines 25 circles numbered from 1 to 25 as quickly as possible.

The Stroop color reading (Stroop, 1935; Meulemans, 2008): Colored rectangles are presented, and participants have to name the colors.

The Stroop word reading (Stroop, 1935; Meulemans, 2008): Participants have to read aloud color names printed in black ink.

The digit span forward task (Wechsler and Naglieri, 2009): Participants are read aloud a sequence of numbers and recall the numbers in the same order.

\section{Assessment of Executive Functions}

The TMT, part B (Reitan, 1958; Godefroy, 2008): Participants are required to connect numbers and letters alternatively as quickly as possible. The TMT part B-part A was also calculated as a measure of cognitive flexibility independent of processing speed.

The Phonemic Verbal Fluency (PVF; Godefroy, 2008): Participants are asked to generate as many words as possible beginning with the letter "P," lasting $2 \mathrm{~min}$.

The Semantic Verbal Fluency (SVF; Godefroy, 2008): Participants are asked to generate as many words as possible belonging to the category "animals," lasting $2 \mathrm{~min}$.

The Alternate Verbal Fluency (AVF; Iudicello et al., 2008): Participants have to continuously alternate words beginning with the letter " $P$ " and words belonging to the category "Animals," lasting $2 \mathrm{~min}$. A shifting index was computed according to the following formula: total words generated in the AVF subtest/[(PVF + SVF score)/2]. This index was used to assess the shifting cost a participant pays passing from performing the single fluency subtests to performing the AVF subtest (Costa et al., 2014).

The Stroop color interference (C/W, Stroop, 1935; Meulemans, 2008): Participants have to name the ink color of a printed word that spells the name of a different color. A Stroop difference score was computed (i.e., C/W - Stroop color read) as a measure of response inhibition independent of processing speed (Meulemans, 2008).

The digit span backward task (Wechsler and Naglieri, 2009): Participants are read aloud a sequence of numbers and recall the numbers in reverse order. A digit difference score was calculated (i.e., digit span forward - digit span backward) as a measure of data manipulation independent of storage information (Wager and Smith, 2003; Ruscheweyh et al., 2013).

The Frontal Assessment Battery (FAB; Dubois et al., 2000): This battery consists of six subtests exploring the following: conceptualization, mental flexibility, motor programming, sensitivity to interference, inhibitory control, and environmental autonomy. In addition to the global score, we also used the Go-No Go task subscore that explores the domain of inhibitory control.

\section{Assessment of Verbal Episodic Memory}

The Free and Cued Selective Reminding Test (FCSRT; Grober and Buschke, 1987; Van der Linden et al., 2004): This test assesses the ability to learn a 16 written word list that refers to 16 semantic categories. This test provided an immediate cued recall score (i.e., encoding phase), a total free recall score (i.e., participants are asked to retrieve the words spontaneously), and a total recall score, which was the sum of free and cued recall (i.e., participants are asked to retrieve the words with the help of a semantic cue). The total number of intrusions (i.e., words absent from the list and falsely recalled) was also recorded.

\section{Apparatus}

Saccadic eye movements were recorded using a remote eyetracking device at a frequency of $250 \mathrm{~Hz}$, an accuracy of $0.4^{\circ}$, and a spatial resolution of $0.03^{\circ}$ (RED 500, $\mathrm{SMI}^{\circledR}$, Teltow, Germany). We used DELL E6530 Laptop with an Intel Core i7 processor and a 22 -inch display screen with a resolution of $1920 \times 1080$ pixels and a refresh rate at $60 \mathrm{~Hz}$.

\section{Saccadic Eye Movement Tasks Prosaccade Task}

Each trial started with a central white fixation point $\left(0.5^{\circ}\right.$ of visual angle) on a black background. After 2,000 ms, a white targetpoint $\left(0.5^{\circ}\right.$ of visual angle) appeared for $2,000 \mathrm{~ms}$. Then a new central fixation-point appeared to signal the start of the next trial. In the step condition, the central dot disappears simultaneously with the target dot appearance. In the gap condition, the central dot disappears $200 \mathrm{~ms}$ before the target dot appears (i.e., "gap" condition). In the overlap condition, the central dot disappears $200 \mathrm{~ms}$ after the target dot appears (Figure 1). Every target-point was displayed with an eccentricity of $\pm 8^{\circ}$ or $\pm 16^{\circ}$ of visual angle in the horizontal plane. Participants were instructed to keep their gaze on the central fixation-point until the peripheral target point appeared and at this time, they had to look at the target point as accurately and quickly as possible.

\section{Antisaccade Task}

The AS task was similar to the PS task except for the instructions given to the participants (see Figure 1). Participants were instructed to keep their gaze on the central fixation-point until 


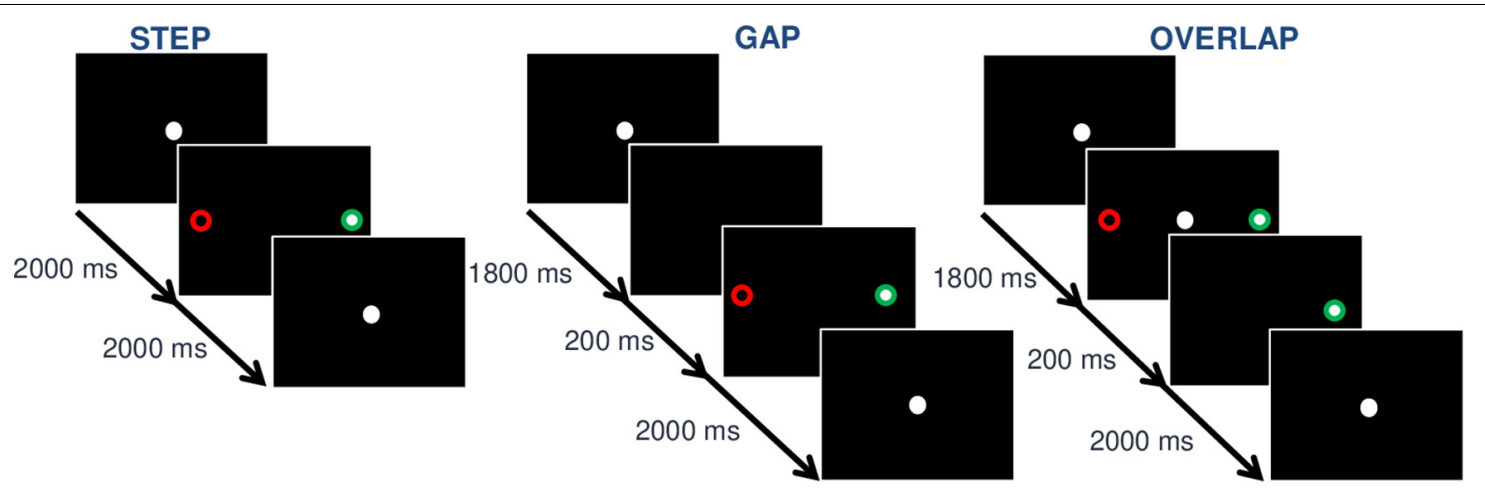

FIGURE 1 | Illustration of the gap, step, and overlap conditions in a paradigm of prosaccade (PS) and antisaccade (AS). The o represents the point displayed, 。 the optimal fixation in the AS task, and o the optimal fixation in PS task. In the step condition (left), the central dot disappears simultaneously with the target dot appearance. In the step condition (left), the central dot simultaneously disappears when the target appears. In the gap condition (middle), the central dot disappears $200 \mathrm{~ms}$ before the target dot appears. In the overlap condition (right), the central dot disappears 200 ms after the target dot appears.

the peripheral target-point appeared. After the target-point onset, they had to direct their gaze in the opposite direction to the target-point as quickly and accurately as possible.

\section{Procedure}

The experiment was divided into two sessions. In the first session, patients underwent a neuropsychological assessment. In the second session, patients performed the SEM tasks. Neuropsychological tests and SEM tasks were performed 1 week apart. At the beginning of the second session, the patient was seated in a quiet room, $60 \mathrm{~cm}$ in front of the monitor, and the eye-tracking system. Each participant completed two blocks of 48 trials. Block orders were counterbalanced between patients (i.e., PS-AS or AS-PS) with a 5-min break and a 5point calibration between each block. The three conditions were randomly distributed in each block (i.e., 16 steps, 16 gaps, and 16 overlaps). Five practice trials for each block were performed to ensure that participants understood instructions.

\section{Data Reduction and Analysis}

Saccade onset and offset were defined by a fixed velocity threshold of $30 \%$ s. The direction of a saccade was determined by the eye position difference between the start and the end of the saccade. Trials containing anticipated saccades (less than $80 \mathrm{~ms}$ ) or delayed saccades (more than $800 \mathrm{~ms}$ ) at the target onset were excluded from the analysis. Trials were also excluded when the eye tracker failed to record the eye coordinates (e.g., eye blink, loss of pupil, or corneal reflection).

Saccades directed toward the targets in the PS task and in the opposite direction in AS task were defined as correct saccades. Saccades directed toward the targets in AS task were defined as AS errors. PS errors were not analyzed because their number was too low for an informative statistical analysis. In AS task, when a subsequent saccade goes in the opposite direction after an AS error, the former was classified as a corrected AS error. Finally, we calculated a gap-effect index [i.e., gap-step ratios: $($ gap - step $) /($ gap + step)] and overlap-effect ratios [i.e., overlap-step ratios: (overlap - step)/(overlap + step)] for each SEM latency just cited above.

As the Shapiro-Wilk's test did not show non-normality distributions and the Levene's test did not show heterogeneity of the variances for all SEM parameters, we decided to use parametric ANOVA. Patients (patients with SB vs. patients with no SB) were the between-subject factor and conditions (gap, step, and overlap) the within-subject factor. When the assumption of sphericity was violated, we used the Greenhouse-Geisser correction.

\section{RESULTS}

\section{Demographic, Clinical, and Neuropsychological Variables}

As indicated by Table 1, participant groups did not differ significantly on demographic variables, such as male/female ratio, age, and years of education. There was no statistically significant difference according to the severity level of depression. There were no statistically significant differences in cognition performance between the two participant groups.

\section{Saccadic Eye Movement Variables Prosaccade Task}

Analysis of variance on PS latency revealed only a main effect of conditions, $F(2,44)=66.09, p<0.001, \eta^{2} p=0.75$. Saccades were faster in gap condition $(M=237.62, \mathrm{SD}=30.01)$ than in step $(M=288.45, \mathrm{SD}=35.19, p<0.001)$ and overlap conditions $(M=326.62, \mathrm{SD}=50.97, p<0.001)$, and they were faster in step than overlap conditions $(p<0.001)$. The main effect of patients $\left[F(1,22)=0.54, p=0.47, \eta^{2} P=0.02\right]$ and the patients $\times$ conditions interaction $[F(2,44)=2.13, p=0.13$, $\eta^{2}{ }_{P}=0.09$ ] were not statistically significant (Figure 2).

As regards the gap-step and the overlap-step ratios, a main effect of conditions was found $[F(1,22)=129.63, p<0.001$, $\left.\eta^{2}{ }_{p}=0.86\right]$. Gap-step ratio $(M=-0.10, \mathrm{SD}=0.06)$ was lower than the overlap-step ratio $(M=0.06, \mathrm{SD}=0.06)$. Gap-step 
TABLE 1 | Demographic, clinical, and neuropsychological characteristics of the study sample.

\begin{tabular}{|c|c|c|c|c|c|}
\hline Variables & SB patients & no SB patients & $t, U$ or $\chi^{2}$ & $p$ & Cohen's d \\
\hline Sex (male/female) & $5 / 7$ & $3 / 9$ & $0.75^{b}$ & 0.39 & \\
\hline Age & $76.25(6.70)$ & $73.00(6.72)$ & 0.67 & 0.51 & 0.27 \\
\hline Education (years) & 7.67 (3.31) & $8.42(3.82)$ & $68.50^{\mathrm{a}}$ & 0.84 & 0.21 \\
\hline MADRS & $28.50(5.13)$ & $25.00(4.31)$ & 1.81 & 0.08 & 0.74 \\
\hline MMSE & $24.75(2.70)$ & $24.92(2.68)$ & -0.15 & 0.88 & 0.06 \\
\hline FCSRT Immediate recall & $13.00(2.80)$ & $14.92(1.44)$ & $42.50^{\mathrm{a}}$ & 0.08 & 0.86 \\
\hline FCSRT Free recall & $19.83(7.63)$ & $26.00(7.43)$ & -2.01 & 0.06 & 0.82 \\
\hline FCSRT Total recall & $41.58(5.87)$ & $44.42(4.62)$ & $43.00^{\mathrm{a}}$ & 0.10 & 0.54 \\
\hline FCSRT Intrusions & $1.83(3.13)$ & $0.75(1.48)$ & $58.00^{\mathrm{a}}$ & 0.38 & 0.44 \\
\hline SVF & $16.42(7.82)$ & $16.00(6.51)$ & 0.14 & 0.89 & 0.06 \\
\hline PVF & $13.33(4.10)$ & $13.25(5.82)$ & 0.04 & 0.97 & 0.02 \\
\hline AVF & $12.3(8.94)$ & $8.83(5.15)$ & 1.18 & 0.25 & 0.48 \\
\hline Shifting Index & $1.11(1.39)$ & $0.62(0.36)$ & 69 & 0.88 & 0.49 \\
\hline TMT A (s) & 66.33 (26.33) & 69.09 (36.95) & $61.00^{a}$ & 0.78 & 0.09 \\
\hline TMT B (s) & $257(47.9)$ & $236(59.1)$ & 0.99 & 0.33 & 0.40 \\
\hline TMT B-A (s) & $202(43.2)$ & $175(45.4)$ & 1.47 & 0.16 & 0.60 \\
\hline Stroop C & 87.42 (25.39) & $79.00(12.07)$ & $48.50^{\mathrm{a}}$ & 0.29 & 0.42 \\
\hline Stroop W & $60.17(15.41)$ & $64.73(25.23)$ & $65.50^{\mathrm{a}}$ & 1.00 & 0.22 \\
\hline Stroop C/W & 206 (72.3) & $182(35.7)$ & 0.94 & 0.36 & 0.38 \\
\hline Stroop C/W - C & $118(55.7)$ & $102(42.0)$ & 0.83 & 0.41 & 0.34 \\
\hline Digit span forward & $4.75(0.87)$ & $4.42(0.79)$ & $55.50^{\mathrm{a}}$ & 0.28 & 0.40 \\
\hline Digit span backward & $3.75(0.97)$ & $3.42(0.67)$ & $58.00^{a}$ & 0.38 & 0.40 \\
\hline Digit difference score & $1.00(0.74)$ & $1.00(0.95)$ & & & \\
\hline FAB total score & $13.75(2.14)$ & $14.67(2.50)$ & -0.97 & 0.35 & 0.39 \\
\hline FAB (Go-No Go) & 1.67 (1.07) & $2.17(1.27)$ & -1.04 & 0.31 & 0.43 \\
\hline
\end{tabular}

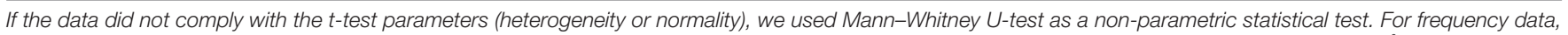

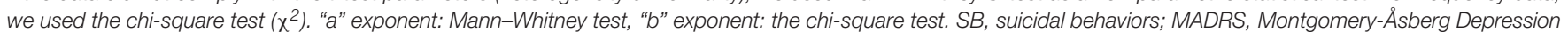

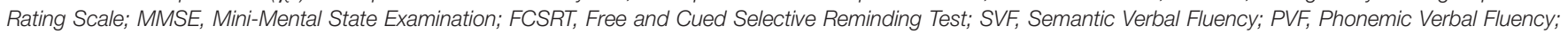

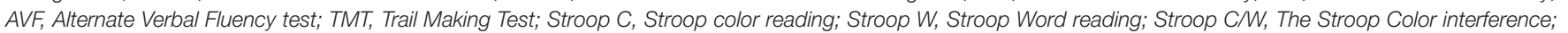
FAB, Frontal Assessment Battery.

ratio and overlap-step ratio were statistically different from zero $[t(23)=-7.71, p<0.001, t(23)=4.47, p<0.001$, respectively] The main effect of patients $\left[F(1,22)=0.52, p=0.48, \eta^{2}{ }_{P}=0.02\right]$ as well as patients $\times$ conditions were not statistically significant (see Figure 2).

\section{Antisaccade Task}

\section{Correct Antisaccade}

We found only a main effect of conditions on correct AS latency, $F(2,44)=28.49, p<0.001, \eta^{2}{ }_{P}=0.56$. Saccades were longer in overlap condition $(M=581.24, \mathrm{SD}=100.86)$ than in step $(M=459.55, \mathrm{SD}=67.93, p<0.001)$ and gap conditions $(M=426.72, \mathrm{SD}=93.88, p<0.001)$. There was no statistical difference between step and gap conditions $(p=0.41)$. The main effect of patients $\left[F(1,22)=1.35, p=0.26, \eta^{2}{ }_{P}=0.06\right]$ and the patients $\times$ conditions interaction $[F(2,44)=0.84, p=0.44$, $\left.\eta^{2} P=0.04\right]$ were not statistically significant.

The gap-step and the overlap-step ratios, a main effect of conditions was found $[F(1,22)=41.25, p<0.001$, $\left.\eta_{p}^{2}=0.65\right]$. Gap-step ratio was $(M=-0.04, \mathrm{SD}=0.12)$ lower than the overlap-step ratio $(M=0.11, \mathrm{SD}=0.10)$. Overlap-step ratio was statistically different from zero $[t(23)=5.59, p<0.001]$, contrary to gap-step ratio $[t(23)=-1.89, p=0.07]$. The main effect of patients
$\left[F(1,22)=0.89, p=0.35, \eta^{2}=0.04\right]$ and patients $\times$ conditions interaction $\left[F(1,22)=0.67, p=0.42, \eta^{2}{ }_{P}=0.03\right]$ were not statistically significant.

As regards the proportion of correct AS, a main effect of condition was found, $F(2,44)=3.96, p=0.03, \eta^{2}{ }_{P}=0.15$. Patients made more correct AS in overlap condition $(M=33.71$, $\mathrm{SD}=5.66)$ than step conditions $(M=24.33, \mathrm{SD}=22.10$, $p=0.04)$. There was no difference between gap $(M=25.96$, $\mathrm{SD}=26.05)$ and overlap $(p=0.09)$ or step conditions $(p=0.89)$. ANOVA neither revealed main effect of patients $[F(1,22)=0.31$, $\left.p=0.58, \eta_{P}^{2}=0.01\right]$ nor patients $\times$ conditions interaction $\left[F(2,44)=2.14, p=0.13, \eta^{2}=0.09\right]$.

\section{Antisaccade Errors}

As regards AS error latency, we found a main effect of conditions, $F(1.38,30.29)=36.47, p<0.001, \eta^{2} P=0.62$. Saccades were faster in gap condition $(M=249.38, \mathrm{SD}=42.40)$ than in step $(M=298.17, \mathrm{SD}=43.29, p=0.004)$ and overlap conditions $(M=352.26, \mathrm{SD}=68.04, p<0.001)$, and they were faster in step than overlap condition $(p<0.001)$. The main effect of patients $\left[F(1,22)=2.04, p=0.17, \eta^{2}{ }_{P}=0.09\right]$ and the patients $\times$ conditions interaction $[F(1.38,30.29)=1.49, p=0.24$, $\left.\eta^{2} P=0.07\right]$ were not statistically significant. 


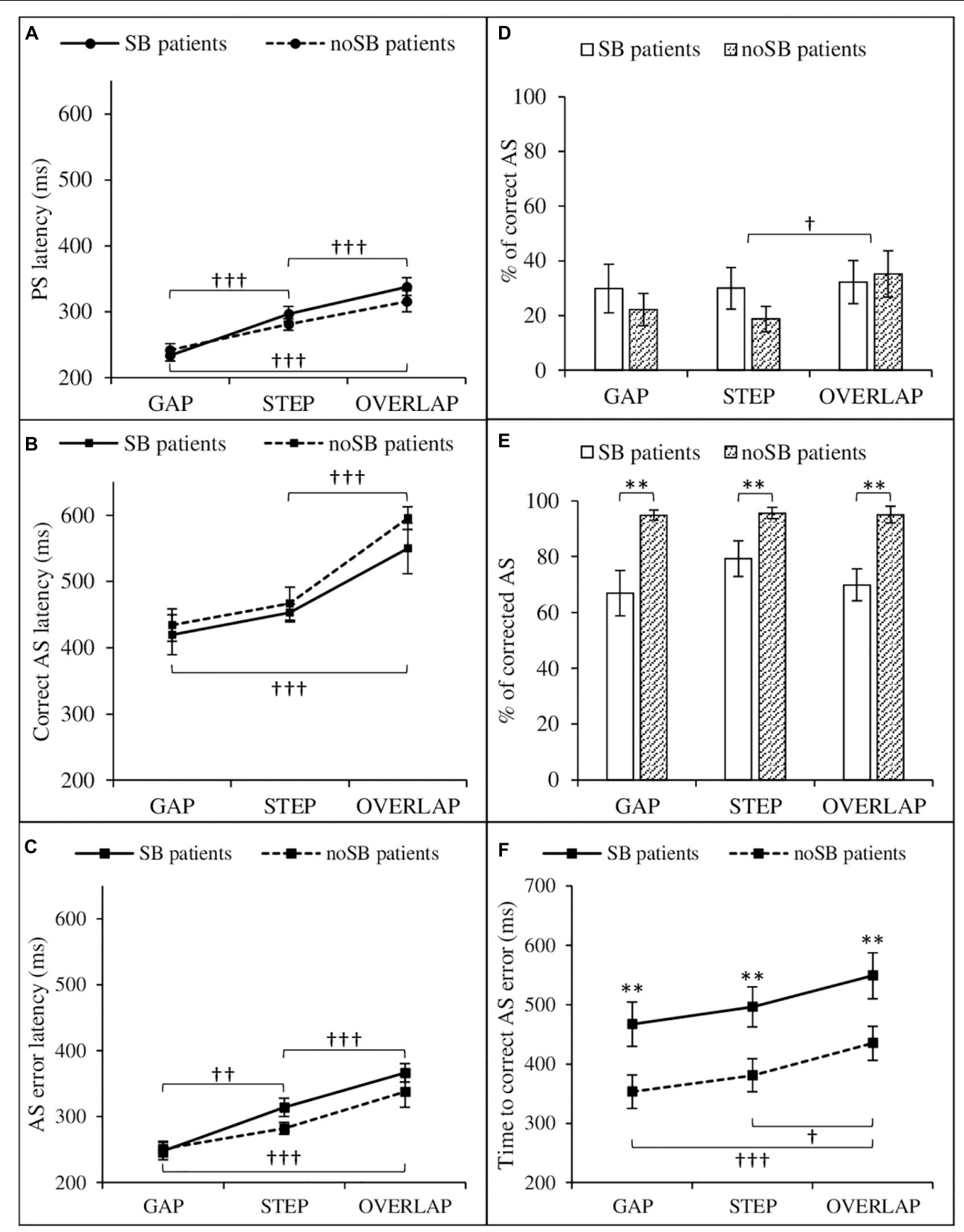

FIGURE 2 | Saccadic eye movement parameters as a function of condition (gap, step, and overlap) and patient group (SB patients and no SB patients). Left: prosaccades (A), correct antisaccades (B), and antisaccade errors (C) latencies. Right: proportion (i.e., percentage of the total number of saccades) of correct antisaccades (D), proportion of corrected antisaccades among antisaccade errors (E), time to correct antisaccade errors (F). AS, antisaccade; no SB, no suicidal behaviors; PS, prosaccade; SB, suicidal behaviors. Error bars: \pm 1 standard errors. Significant differences between condition are represented by " ${ }^{\dagger}$ " ${ }^{\dagger} p<0.05$; $\left.{ }^{\dagger \dagger} p<0.01 ;{ }^{\dagger \dagger} p<0.001\right)$. Significant differences between patients with SB and patients with no SB are represented by "*" $(* * p<0.01)$.

The gap-step and the overlap-step ratios analysis showed a main effect of conditions $[F(1,22)=63.88, p<0.001$, $\left.\eta_{p}^{2}=0.74\right]$; gap-step ratio $(M=-0.10, \mathrm{SD}=0.07)$ was lower than the overlap-step ratio $(M=0.10, \mathrm{SD}=0.12)$. Gap-step ratio and overlap-step ratio were statistically different from zero $[t(23)=-7.34, p<0.001, t(23)=4.30$, $p<0.001$, respectively]. The main effect of patients $\left[F(1,22)=0.31, p=0.59, \eta^{2} P=0.01\right]$ and patients $\times$ conditions interaction $\left[F(1,22)=0.85, p=0.37, \eta^{2} P=0.04\right]$ were not statistically significant.

Analysis of variance on the proportion of corrected errors among the total of AS errors revealed only a main effect of patients $\left[F(1,22)=13.00, p=0.002, \eta^{2}{ }_{P}=0.37\right]$. Patients with SB made less corrections $(M=72.03, \mathrm{SD}=24.43)$ than patients with no $\mathrm{SB}(M=95.13, \mathrm{SD}=7.95)$. The main effect of conditions $\left[F(1.48,32.56)=3.02, p=0.08, \eta^{2} P=0.12\right]$, and the 
patients conditions interaction $[F(1.48,32.56)=2.35, p=0.12$, $\left.\eta^{2} P=0.10\right]$ did not reach statistical significance.

Finally, concerning the time to correct AS errors, we found a main effect of patients $[F(2,44)=8.99, p<0.001$, $\left.\eta^{2} P=0.29\right]$. Patients with SB took more time to correct AS errors $(M=504.09, \mathrm{SD}=128.00)$ than patients with no $\mathrm{SB}$ $(M=384.82, \mathrm{SD}=95.43)$. A main effect of conditions was also found $\left[F(1,22)=9.46, p=0.006, \eta^{2} P=0.30\right]$. The time to correct AS errors was longer in overlap condition $(M=490.80$, $\mathrm{SD}=126.82)$ than in gap $(M=407.86, \mathrm{SD}=125.02$, $p<0.001)$ and step conditions $(M=434.69, \mathrm{SD}=120.29$, $p=0.02)$. There was no statistical difference between gap and step conditions $(p=0.56)$. Patients $\times$ conditions interaction did not reach statistical significance $[F(1,22)=0.02, p=0.98$, $\left.\eta^{2}{ }_{P}=0.001\right]$.

\section{DISCUSSION}

In this study, oculomotor impairments were found in elderly patients with SB. In AS tasks, although patients with SB and no SB had a similar proportion of correct AS, patients with SB had fewer corrected AS errors and they took more time to correct them than patients with no SB. Our results are consistent with the literature since Noiret et al. (2017) have reported a link between oculomotor impairments and inhibitory and cognitive flexibility measures in healthy elderly while other studies have found cognitive inflexibility among suicidal elderly (Neuringer, 1964; Richard-Devantoy et al., 2013). Our results suggested higher cognitive inflexibility in suicidal patients compared to non-suicidal. Cognitive flexibility can be defined as the ability to adapt cognitive processing strategies to face new and unexpected conditions in the environment (Cañas et al., 2003). Flexibility function impairment could explain the difficulty of depressed elderly with SB in generating solutions to the resurgence of SI to respond adequately to stressful environments as well as the difficulty to correct their errors in AS task.

Moreover, both groups had similar performances for PS latency. This result suggests that patients with SB do not result in a more pronounced decline in processing speed affecting saccade triggering than patients with no SB. Regardless of the groups, our study showed an increase in PS, correct AS and AS error latencies in overlap conditions compared to gap and step conditions. These results are in agreement with reported literature about gap and overlap effect (Saslow, 1967; Fischer and Boch, 1983; Kalesnykas and Hallett, 1987; Braun and Breitmeyer, 1988; Fischer et al., 1993; Crevits and Vandierendonck, 2005; Kristjansson, 2007; Noiret et al., 2017). Overlap condition requires attentional disengagement of the dot, which may explain higher saccadic latency than in gap and step conditions. Our results suggest a similar disengagement process and attentional capture for both patient groups in PS and AS tasks.

Some studies (Keilp et al., 2008, 2013) have shown that poorer performance on the Stroop task suggested a relatively independent marker of suicide risk within the context of depression. However, in our study, the neuropsychological assessment not only showed similar results between both groups in term of attention, processing speed, verbal episodic memory but also EF. These results support the idea that the SEM might be a more relevant tool for studying SB in the elderly. In our experiment, simple measures, such as reaction time (i.e., latency) or correct/incorrect trial, were failed to differentiate between patient groups. However, the precision and the continuous recording of the data during trials had produced finer variables (i.e., proportion of corrected errors and time to correct errors). These variables showed that correcting errors is more difficult for patients with SB than for patients with no SB.

A possible limitation of this study is related to the effect of drugs on SEMs. Some studies showed that drugs can affect eye movements (Wells et al., 2014), while others did not show any treatment effect on eye movements in depressed patients (Katsanis et al., 1997; Flechtner et al., 2002). To reduce the effects of the drugs, all of our patients took psychotropic drugs from the same family and were in a stable phase of their disease. Another limitation of this study was the small number of participants due to the difficulty to enroll this type of population. Furthermore, we used a convenience sample of inpatients that may not be representative of community-dwelling older adults. A larger sample size using randomly recruited representative samples should be needed to replicate our findings and further specifying SEM characteristics in patients with SB.

Our main findings showed that elderly depressed patients with SB had difficulties in correcting errors in AS task in comparison with elderly depressed patients with no SB. The assessment of eye movement parameters in depressed elderly patients with SB maybe a first step in identifying patients at risk. Our results are hopeful and encourage further research to confirm the interest in using eye movement measurements as a complementary tool for $\mathrm{EF}$ evaluation and to predict suicidal risks in the elderly patients.

\section{DATA AVAILABILITY STATEMENT}

The raw data supporting the conclusions of this article will be made available by the authors, without undue reservation.

\section{ETHICS STATEMENT}

The studies involving human participants were reviewed and approved by the Committee for the Protection of Persons (CPP) and was conducted in accordance with declaration of Helsinki Declaration as revised in 1989. The patients/participants provided their written informed consent to participate in this study.

\section{AUTHOR CONTRIBUTIONS}

YB: study concept and design, methodology, analysis and interpretation of the data, preparation of the manuscript, and revising the manuscript. NN: study concept and design, statistical analysis and interpretation of the data, methodology, and revising the manuscript. BL: analysis interpretation and acquisition of the data. JM: funding acquisition and project administration. 
CD: acquisition of the data. JH: patient enrollment. CMa and DB: study concept and design. SR-D, CMo, EH, and EL: study concept and design and funding acquisition. PV: study concept and design, funding acquisition, project administration, patient enrollment, and revising the manuscript. GC: study concept and design, methodology, and revising the manuscript. All authors contributed to the article and approved the submitted version.

\section{REFERENCES}

Alexopoulos, G. S., Bruce, M. L., Hull, J., Sirey, J. A., and Kakuma, T. (1999). Clinical determinants of suicidalf ideation and behavior in geriatric depression. Arch. Gen. Psychiatry 56, 1048-1053. doi: 10.1001/archpsyc.56.11.1048

American Psychiatric Association (2013). Diagnostic and Statistical Manual of Mental Disorders (DSM-5\$). Washington, DC: American Psychiatric Association.

Armstrong, T., and Olatunji, B. O. (2012). Eye tracking of attention in the affective disorders: a meta-analytic review and synthesis. Clin. Psychol. Rev. 32, 704-723. doi: 10.1016/j.cpr.2012.09.004

Barsznica, Y., Noiret, N., Chopard, G., and Vandel, P. (2019). Potential interest of ocular saccades for detection of suicidal behavior in depressed elderly. Geriatr. Psychol. Neuropsychiatr. Vieil. 17, 92-98. doi: 10.1684/pnv.2019.0785

Bazin, N. (2004). Conduites suicidaires et dépression du sujet âgé. Psychol. Neuropsychiatr. Vieil. 2, 29-33.

Braun, D., and Breitmeyer, B. (1988). Relationship between directed visual attention and saccadic reaction times. Exp. Brain Res. 73, 546-552. doi: 10. 1007/BF00406613

Cañas, J., Quesada, J., Antolí, A., and Fajardo, I. (2003). Cognitive flexibility and adaptability to environmental changes in dynamic complex problem-solving tasks. Ergonomics 46, 482-501. doi: 10.1080/0014013031000061640

Carvalho, N., Noiret, N., Vandel, P., Monnin, J., Chopard, G., and Laurent, E. (2014). Saccadic eye movements in depressed elderly patients. PLoS One 9:e105355. doi: 10.1371/journal.pone.0105355

Conwell, Y., and Thompson, C. (2008). Suicidal behavior in elders. Psychiatr. Clin. N. Am. 31, 333-356. doi: 10.1016/j.psc.2008.01.004

Costa, A., Bagoj, E., Monaco, M., Zabberoni, S., De Rosa, S., Papantonio, A. M., et al. (2014). Standardization and normative data obtained in the Italian population for a new verbal fluency instrument, the phonemic/semantic alternate fluency test. Neurol. Sci. 35, 365-372. doi: 10.1007/s10072-013-1520-8

Crevits, L., and Vandierendonck, A. (2005). Gap effect in reflexive and intentional prosaccades. Neuropsychobiology 51, 39-44. doi: 10.1159/000082854

De Leo, D., Padoani, W., Scocco, P., Lie, D., Bille-Brahe, U., Arensman, E., et al. (2001). Attempted and completed suicide in older subjects: results from the WHO/EURO multicentre study of suicidal behaviour. Int. J. Geriatr. Psychiatry 16, 300-310. doi: 10.1002/gps.337

Dubois, B., Slachevsky, A., Litvan, I., and Pillon, B. (2000). The FAB: a frontal assessment battery at bedside. Neurology 55, 1621-1626. doi: 10.1212/WNL. 55.11 .1621

Everling, S., and Fischer, B. (1998). The antisaccade: a review of basic research and clinical studies. Neuropsychologia 36, 885-899. doi: 10.1016/S0028-3932(98) 00020-7

Fischer, B., and Boch, R. (1983). Saccadic eye movements after extremely short reaction times in the monkey. Brain Res. 260, 21-26. doi: 10.1016/00068993(83)90760-6

Fischer, B., Weber, H., Biscaldi, M., Aiple, F., Otto, P., and Stuhr, V. (1993). Separate populations of visually guided saccades in humans: reaction times and amplitudes. Exp. Brain Res. 92, 528-541. doi: 10.1007/BF00229043

Flechtner, K.-M., Steinacher, B., Sauer, R., and Mackert, A. (2002). Smooth pursuit eye movements of patients with schizophrenia and affective disorder during clinical treatment. Eur. Arch. Psychiatry Clin. Neurosci. 252, 49-53. doi: 10. 1007/s004060200011

Folstein, M. F., Folstein, S. E., and Mchugh, P. R. (1975). "Mini-mental state": a practical method for grading the cognitive state of patients for the clinician. J. Psychiatr. Res. 12, 189-198. doi: 10.1016/0022-3956(75)90026-6

Godefroy, O. (2008). Fonctions Exécutives et Pathologies Neurologiques et Psychiatriques: Évaluation en Pratique Clinique. Marseille: Solal.

\section{FUNDING}

This research was supported jointly by the research mission of the Research, Studies, Evaluation, and Statistics (Mire-DREES), Ministry of Health (DGS), and National Health Insurance Fund for Salaried Workers (CNAMTS), as part of the call for research launched by IRESP in 2015 .

Grober, E., and Buschke, H. (1987). Genuine memory deficits in dementia. Dev. Neuropsychol. 3, 13-36. doi: 10.1080/87565648709540361

Hutton, S. B., and Ettinger, U. (2006). The antisaccade task as a research tool in psychopathology: a critical review. Psychophysiology 43, 302-313. doi: 10.1111/ j.1469-8986.2006.00403.x

Iudicello, J. E., Woods, S. P., Weber, E., Dawson, M. S., Scott, J. C., Carey, C. L., et al. (2008). Cognitive mechanisms of switching in HIV-associated category fluency deficits. J. Clin. Exp. Neuropsychol. 30, 797-804. doi: 10.1080/ 13803390701779578

Iverson, G. L., Brooks, B. L., Langenecker, S. A., and Young, A. H. (2011). Identifying a cognitive impairment subgroup in adults with mood disorders. J. Affect. Disord. 132, 360-367. doi: 10.1016/j.jad.2011.03.001

Jurado, M. B., and Rosselli, M. (2007). The elusive nature of executive functions: a review of our current understanding. Neuropsychol. Rev. 17, 213-233. doi: 10.1007/s11065-007-9040-z

Kalafat, M., Hugonot-Diener, L., and Poitrenaud, J. (2003). Standardisation et étalonnage français du "mini mental state"(MMS) version GRECO. Rev. Neuropsychol. 13, 209-236.

Kalesnykas, R., and Hallett, P. (1987). The differentiation of visually guided and anticipatory saccades in gap and overlap paradigms. Exp. Brain Res. 68, 115-121. doi: 10.1007/BF00255238

Katsanis, J., Kortenkamp, S., Iacono, W. G., and Grove, W. M. (1997). Antisaccade performance in patients with schizophrenia and affective disorder. J. Abnorm. Psychol. 106, 468-472. doi: 10.1037/0021-843X.106.3.468

Keilp, J., Gorlyn, M., Russell, M., Oquendo, M., Burke, A., Harkavy-Friedman, J., et al. (2013). Neuropsychological function and suicidal behavior: attention control, memory and executive dysfunction in suicide attempt. Psychol. Med. 43, 539-551. doi: 10.1017/S0033291712001419

Keilp, J. G., Gorlyn, M., Oquendo, M. A., Burke, A. K., and Mann, J. J. (2008). Attention deficit in depressed suicide attempters. Psychiatry Res. 159, 7-17. doi: 10.1016/j.psychres.2007.08.020

King, D. A., Conwell, Y., Cox, C., Henderson, R. E., Denning, D. G., and Caine, E. D. (2000). A neuropsychological comparison of depressed suicide attempters and nonattempters. J. Neuropsychiatry Clin. Neurosci. 12, 64-70. doi: 10.1176/ jnp.12.1.64

Kristjansson, A. (2007). Saccade landing point selection and the competition account of pro-and antisaccade generation: the involvement of visual attentionA review. Scand. J. Psychol. 48, 97-113. doi: 10.1111/j.1467-9450.2007.0 0537.x

Leigh, R. J., and Kennard, C. (2004). Using saccades as a research tool in the clinical neurosciences. Brain 127, 460-477. doi: 10.1093/brain/awh035

Levy, N. K., Lavidor, M., and Vakil, E. (2018). Prosaccade and antisaccade paradigms in persons with Alzheimer's disease: a meta-analytic review. Neuropsychol. Rev. 28, 16-31. doi: 10.1007/s11065-017-9362-4

Marzuk, P. M., Hartwell, N., Leon, A., and Portera, L. (2005). Executive functioning in depressed patients with suicidal ideation. Acta Psychiatr. Scand. 112, 294301. doi: 10.1111/j.1600-0447.2005.00585.x

Mcgirr, A., Dombrovski, A. Y., Butters, M. A., Clark, L., and Szanto, K. (2012). Deterministic learning and attempted suicide among older depressed individuals: cognitive assessment using the wisconsin card sorting task. J. Psychiatr. Res. 46, 226-232. doi: 10.1016/j.jpsychires.2011.10.001

Meulemans, T. (2008). “La batterie GREFEX," in Fonctions Executives et Pathologies Neurologiques et Psychiatriques, ed. Deboeck (Marseille: Solal), 217-229. doi: 10.1080/13825585.2016.1226248

Miyake, A., Friedman, N. P., Emerson, M. J., Witzki, A. H., Howerter, A., and Wager, T. D. (2000). The unity and diversity of executive functions and their contributions to complex "Frontal Lobe" tasks: a latent variable analysis. Cogn. Psychol. 41, 49-100. doi: 10.1006/cogp.1999.0734 
Montgomery, S. A., and Asberg, M. (1979). A new depression scale designed to be sensitive to change. Br. J. Psychiatry 134, 382-389. doi: 10.1192/bjp.134.4.382

Neuringer, C. (1964). Rigid thinking in suicidal individuals. J. Consult. Clin. Psychol. 28, 54-58. doi: 10.1037/h0045809

Noiret, N., Vigneron, B., Diogo, M., Vandel, P., and Laurent, É (2017). Saccadic eye movements: what do they tell us about aging cognition? Aging Neuropsychol. Cogn. 24, 575-599. doi: 10.1080/13825585.2016.1237613

Posner, K., Brent, D., Lucas, C., Gould, M., Stanley, B., Brown, G., et al. (2008). Columbia-Suicide Severity Rating Scale (C-SSRS). New York, NY: Columbia University Medical Center.

Pratt, J., Dodd, M., and Welsh, T. (2006). Growing older does not always mean moving slower: examining aging and the saccadic motor system. J. Mot. Behav. 38, 373-382. doi: 10.3200/JMBR.38.5.373-382

Reitan, R. M. (1958). Validity of the trail making test as an indicator of organic brain damage. Percept. Mot. Skill 8, 271-276. doi: 10.2466/pms.1958. 8.3.271

Richard-Devantoy, S., Jollant, F., Deguigne, F., and Letourneau, G. (2013). Neurocognitive markers of suicide vulnerability in the elderly: a review. Geriatr. Psychol. Neuropsychiatr. Vieil. 11, 367-378. doi: 10.1684/pnv.201 3.0442

Richard-Devantoy, S., Jollant, F., Kefi, Z., Turecki, G., Olie, J. P., Annweiler, C., et al. (2012). Deficit of cognitive inhibition in depressed elderly: a neurocognitive marker of suicidal risk. J. Affect. Disord. 140, 193-199. doi: 10.1016/j.jad.2012.03.006

Richard-Devantoy, S., Szanto, K., Butters, M. A., Kalkus, J., and Dombrovski, A. Y. (2015). Cognitive inhibition in older high-lethality suicide attempters. Int. J. Geriatr. Psychiatry 30, 274-283. doi: 10.1002/gps.4138

Richard-Devantoy, S., Turecki, G., and Jollant, F. (2016). Neurobiology of elderly suicide. Arch. Suicide Res. 20, 291-313. doi: 10.1080/13811118.2015.1048397

Ruscheweyh, R., Deppe, M., Lohmann, H., Wersching, H., Korsukewitz, C., Duning, T., et al. (2013). Executive performance is related to regional gray matter volume in healthy older individuals. Hum. Brain Mapp. 34, 3333-3346. doi: $10.1002 / \mathrm{hbm} .22146$

Saslow, M. (1967). Effects of components of displacement-step stimuli upon latency for saccadic eye movement. J. Opt. Soc. Am. 57, 1024-1029. doi: 10. 1364/JOSA.57.001024

Shah, A., Bhat, R., Zarate-Escudero, S., Deleo, D., and Erlangsen, A. (2016). Suicide rates in five-year age-bands after the age of 60 years: the international landscape. Aging Ment. Health 20, 131-138. doi: 10.1080/13607863.2015.1055552

Stroop, J. R. (1935). Studies of interference in serial verbal reactions. J. Exp. Psychol. 18, 643-662. doi: 10.1037/h0054651
Szanto, K., Mulsant, B. H., Houck, P. R., Miller, M. D., Mazumdar, S., and Reynolds, C. F. III (2001). Treatment outcome in suicidal vs. non-suicidal elderly patients. Am. J. Geriatr. Psychiatry 9, 261-268. doi: 10.1097/00019442-200108000-00010

Van der Linden, M., Coyette, F., Poitrenaud, J., Kalafat, M., Calicis, F., Wyns, C., et al. (2004). "Lépreuve de rappel libre/rappel indicé à 16 items (RL/RI-16)," in L'évaluation des Troubles de la Mémoire: Présentation de Quatre Tests de Mémoire Épisodique (avec leur Étalonnage), eds S. A. Van Der Liden, A. Agniel, and C. Baisset Mouly et les membres du GREMEM (Marseille: Solal), 25-47.

Wager, T. D., and Smith, E. E. (2003). Neuroimaging studies of working memory. Cogn. Affect. Behav. Neurosci. 3, 255-274. doi: 10.3758/CABN.3.4.255

Wechsler, D., and Naglieri, J. A. (2009). WNV: Échelle Non Verbale Dintelligence: Manuel. Paris: les Éd. du Centre de psychologie appliquée.

Wells, T. T., Clerkin, E. M., Ellis, A. J., and Beevers, C. G. (2014). Effect of antidepressant medication use on emotional information processing in major depression. Am. J. Psychiatry 171, 195-200. doi: 10.1176/appi.ajp.2013. 12091243

Westheide, J., Quednow, B. B., Kuhn, K.-U., Hoppe, C., Cooper-Mahkorn, D., Hawellek, B., et al. (2008). Executive performance of depressed suicide attempters: the role of suicidal ideation. Eur. Arch. Psychiatry Clin. Neurosci. 258, 414-421. doi: 10.1007/s00406-008-0811-1

World Health Organization (2014). Preventing Suicide: A Global Imperative. Geneva: World Health Organization.

Conflict of Interest: The authors declare that the research was conducted in the absence of any commercial or financial relationships that could be construed as a potential conflict of interest.

Publisher's Note: All claims expressed in this article are solely those of the authors and do not necessarily represent those of their affiliated organizations, or those of the publisher, the editors and the reviewers. Any product that may be evaluated in this article, or claim that may be made by its manufacturer, is not guaranteed or endorsed by the publisher.

Copyright (c) 2021 Barsznica, Noiret, Lambert, Monnin, De Pinho, Hickel, Masse, Richard-Devantoy, Morgny, Bennabi, Haffen, Laurent, Vandel and Chopard. This is an open-access article distributed under the terms of the Creative Commons Attribution License (CC BY). The use, distribution or reproduction in other forums is permitted, provided the original author(s) and the copyright owner(s) are credited and that the original publication in this journal is cited, in accordance with accepted academic practice. No use, distribution or reproduction is permitted which does not comply with these terms. 